\title{
Ultrafast Carrier Dynamics in Exfoliated and Functionalized Calcium Niobate Nanosheets in Water and Methanol
}

\author{
Elizabeth C. Carroll, Owen C. Compton, Dorte Madsen, Frank E. Osterloh, and \\ Delmar S. Larsen* \\ Department of Chemistry, University of California, Davis, One Shields Avenue, Davis, California 95616
}

Received: September 14, 2007; In Final Form: November 9, 2007

\begin{abstract}
We have characterized the initial charge carrier trapping and recombination processes in suspensions of exfoliated calcium niobate nanosheets derived from the Dion Jacobsen phase $\mathrm{KCa}_{2} \mathrm{Nb}_{3} \mathrm{O}_{10}$. Niobate nanosheets have previously been found to catalyze hydrogen generation in photochemical water-splitting reactions. Photogenerated charge carriers relaxed with second-order kinetics on a sub-nanosecond time scale that depended on the nanosheet size. Methanol was used as a sacrificial electron donor to separate the electron and hole dynamics, and the photo-oxidation dynamics was found to directly compete with electron-hole recombination. Absorption spectra for trapped carriers were determined and used to separately analyze the trapped electron and hole dynamics in $\left[\mathrm{H}_{1-x} \mathrm{Ca}_{2} \mathrm{Nb}_{3} \mathrm{O}_{10}\right]^{x-}(x=0.15-0.20)$ sheets in water. Surface modification by 3-aminopropyl(trimethoxy)silane was found to quench trapped carrier absorptions, resulting in an observed optical gain.
\end{abstract}

\section{Introduction}

Ion-exchangeable layered perovskites have generated interest as potential photocatalytic materials capable of $\mathrm{H}_{2}$ generation from pure water. ${ }^{1-10}$ A quantum efficiency for $\mathrm{H}_{2}$ generation of up to $20 \%$ has been observed in $\mathrm{K}_{4} \mathrm{Nb}_{6} \mathrm{O}_{17}$ under $\mathrm{UV}$ irradiation without the aid of cocatalysts or sacrificial donors. ${ }^{11}$ Layered semiconductors can be intercalated with sensitizers for visible light absorption or cocatalysts to improve catalytic activity. ${ }^{12-20}$ Recent work in our lab has shown that the derivatization of individually dispersed (TBA, $\mathrm{H}) \mathrm{Ca}_{2} \mathrm{Nb}_{3} \mathrm{O}_{10}$ nanosheets with $\mathrm{Pt}$ nanoparticles resulted in quantum efficiency for $\mathrm{H}_{2}$ generation of $7.5 \%$ under UV irradiation. ${ }^{21}$

Dispersed niobate sheets can serve as nanoscale templates for self-assembled nanostructures. ${ }^{22-25} \mathrm{We}$ recently showed that sheets derived from $\mathrm{KCa}_{2} \mathrm{Nb}_{3} \mathrm{O}_{10}$ can be functionalized with 3-aminopropyl(trimethoxy)silane (APS), in which silicon is thought to covalently bond to oxygen on the niobate surface. ${ }^{23}$ The other end of APS has a positively charged $\mathrm{NH}_{3}{ }^{+}$group to which negatively charged ligand-capped metal or semiconductor nanoparticles can be electrostatically attached. The ability to link inorganic nanoparticles is a strong asset in designing multicomponent photocatalysts in which the cocatalysts are selected to optimize reduction and oxidation dynamics and interparticle charge transfer. Self-assembled nanostructures of even moderate efficiency may be commercially viable since their manufacturing process can be easily scaled to large quantities. However, chemical modification of semiconductor surfaces can significantly change excited-state dynamics, which is particularly important in photocatalysts where activity is sensitive to surface recombination.

The objective of this study was to characterize carrier trapping and recombination dynamics in exfoliated $\left[\mathrm{H}_{1-x} \mathrm{Ca}_{2} \mathrm{Nb}_{3} \mathrm{O}_{10}\right]^{x-}$ nanosheets $(x=0.15-0.20)$ and APS-functionalized (TBA,H)$\mathrm{Ca}_{2} \mathrm{Nb}_{3} \mathrm{O}_{10}$. We monitored the initial charge carrier trapping and

* Corresponding author. Fax: (530) 752-8995; e-mail: dlarsen@chem. ucdavis.edu recombination processes in individually dispersed nanosheets using femtosecond transient absorption spectroscopy. Ultrafast carrier dynamics has been characterized in related perovskite materials in bulk and restacked sheets, ${ }^{26}$ but exfoliated sheets in solution are not yet well-studied.

The photodynamics of $\left[\mathrm{H}_{1-x} \mathrm{Ca}_{2} \mathrm{Nb}_{3} \mathrm{O}_{10}\right]^{x-}$ sheets has been characterized in both water and methanol solutions. Methanol is known as an efficient sacrificial electron donor for layered metal oxide semiconductors. ${ }^{2}$ In $\mathrm{TiO}_{2}$, a similar inorganic photocatalyst material, methanol is thought to adsorb to surface oxygen vacancies as a methoxy group that can capture holes on a sub-nanosecond time scale. ${ }^{27,28} \mathrm{KCa}_{2} \mathrm{Nb}_{3} \mathrm{O}_{10}$ is known to be catalytically very active for methanol oxidation ${ }^{1,29}$ and, as shown in this study, exhibits hole capture on a sub-100 ps time scale. As a result of methanol oxidation, electrons and holes undergo different dynamics even in the presence of recombination kinetics. The visible wavelength spectra of the trapped charge carriers are identified based on their distinct transient evolution. These carrier spectra are used to separate trapped electron and hole dynamics in $\left[\mathrm{H}_{1-x} \mathrm{Ca}_{2} \mathrm{Nb}_{3} \mathrm{O}_{10}\right]^{x-}$ sheets in water.

\section{Experimental Procedures}

2.1. Materials. $\mathrm{TBA}_{x}\left[\mathrm{H}_{1-x} \mathrm{Ca}_{2} \mathrm{Nb}_{3} \mathrm{O}_{10}\right]$ nanosheets $(x=$ $0.15-0.20$, TBA is tetrabutylammonium) were synthesized according to literature procedures. ${ }^{30,31}$ Water was purified to a resistivity of $>18 \mathrm{M} \Omega$ with a Nanopure II system. Complete exfoliation was established with TEM images collected using a Philips CM120 transmission electron microscope. The lateral dimensions of the exfoliated sheets were controlled by intense sonication with a Sonics Vibracell VC130 probe sonicator. Nanosheets were functionalized with 3-aminopropyl(trimethoxy)silane (APS, Gelest) as previously described. ${ }^{23}$

The nanosheets were stored in water at $\mathrm{pH} 11.5$ to prevent restacking. Prior to optical measurements, the (TBA, $\mathrm{H}) \mathrm{Ca}_{2}-$ $\mathrm{Nb}_{3} \mathrm{O}_{10}$ sheets were rinsed and diluted with water to $\mathrm{pH} 10.5$. 
Aqueous methanol suspensions were prepared with equal niobate concentrations using spectroscopic grade methanol (Aldrich, $>99 \%$ ).

2.2. Transient Absorption Setup. The dispersed-probe transient absorption setup was based on an amplified Ti:sapphire laser system (Spectra Physics Spitfire Pro + Tsunami) producing $2.25-\mathrm{mJ}$ pulses at $800 \mathrm{~nm}$ with $40-\mathrm{fs}$ (fwhm) duration and $1-\mathrm{kHz}$ repetition rate. The niobate nanosheets were excited with 305nm ultraviolet pulses generated as the second harmonic of a visible-wavelength home-built non-collinear optical parametric amplifier (NOPA). The NOPA produced $10-\mu \mathrm{J} 30$-fs pulses at $610 \mathrm{~nm}$. The visible absorption signal due to photogenerated charge carriers was monitored with an ultrafast white light continuum $(400-700 \mathrm{~nm})$. The probe pulse was generated by focusing 30-nJ 800-nm pulses into a 3-mm disk of $\mathrm{CaF}_{2}$, which was slowly translated to prevent burning and to maintain a single-filament continuum. The probe was variably delayed with respect to the pump pulse, and a maximum time delay of $\sim 8$ ns was achieved by quadruple passing the 800 -nm light on a 0.8-m computer-controlled translation state (Newport). The white light was linearly polarized at magic angle $\left(54.7^{\circ}\right)$ with respect to the pump and focused into the sample with reflective optics to minimize pulse dispersion. The transmitted probe light was spectrally dispersed onto a 256-pixel (Hamamatsu S3901) 16-bit diode array (UEI) read on a shot-by-shot basis while the pump light was optically chopped at $500 \mathrm{~Hz}$ to alternately obtain spectra with and without pump exposure. At each time delay, 1000 pumped/unpumped pairs of spectra were collected. Individual pairs lying more than two standard deviations from the average value were discarded prior to calculating the transient spectrum $\Delta \mathrm{OD}=-\log \left(I(\lambda)_{\text {pumped }} / I(\lambda)_{\text {unpumped }}\right)$.

In each experiment, a suspension of dispersed (TBA, $\mathrm{H}) \mathrm{Ca}_{2}-$ $\mathrm{Nb}_{3} \mathrm{O}_{10}$ nanosheets was flowed through a $0.5-\mathrm{mm}$ path length quartz cell (Starna Industries) with an optical density of 0.45 \pm 0.05 at $305 \mathrm{~nm}$. An instrument response function varying between 220 and 240 fs was determined by cross-correlation of the pump and probe pulses measured by two-photon absorption in methanol. The temporal resolution of the experiment was limited by group velocity mismatch between the pump and the probe pulses and cross-phase modulation. In the powerdependence study, the pump energy was varied between 80 and $500 \mathrm{~nJ}$ by attenuating with a variable ND filter, corresponding to fluences in the range of $0.1-0.7 \mathrm{~W} / \mathrm{cm}^{2}$ per pulse.

Emission spectra were collected using a Jobin-Yvon Fluoromax-P fluorimeter. Steady-state absorption spectra were measured with a Shimadzu UV-1700 UV-vis spectrophotometer.

\section{Results}

3.1. Steady-State Characterization. The niobate nanosheets consisted of three layers of corner-shared $\mathrm{NbO}_{6}$ octahedra with $\mathrm{Ca}^{2+}$ ions in the $\mathrm{O}$ voids (Figure $1 \mathrm{C}$, inset). As synthesized, the nanosheets were negatively charged with $15-20 \%$ of protons replaced with tetrabutylammonium counterions. Under these conditions, the sheets occurred as individual particles with a flat morphology and edges that were mostly irregularly shaped and occasionally straight (Figure 1A). Edge lengths ranged from 0.2 to $1.8 \mu \mathrm{m}$ with a mean value of $1.2 \mu \mathrm{m}$. The thickness of the sheets was $1.16 \mathrm{~nm}$, from crystallographic data. ${ }^{30}$ Following $30 \mathrm{~min}$ of ultrasonication, the average nanosheet edge length was reduced to $80 \mathrm{~nm}$ (Figure 1B) with a narrower size distribution $( \pm 50 \mathrm{~nm})$.

Transmission UV-vis spectra of exfoliated (TBA, $\mathrm{H}) \mathrm{Ca}_{2}-$ $\mathrm{Nb}_{3} \mathrm{O}_{10}$ sheets in water at $\mathrm{pH} 10.5$ are shown in Figure 1C. Rayleigh scattering contributed significantly to the absorption
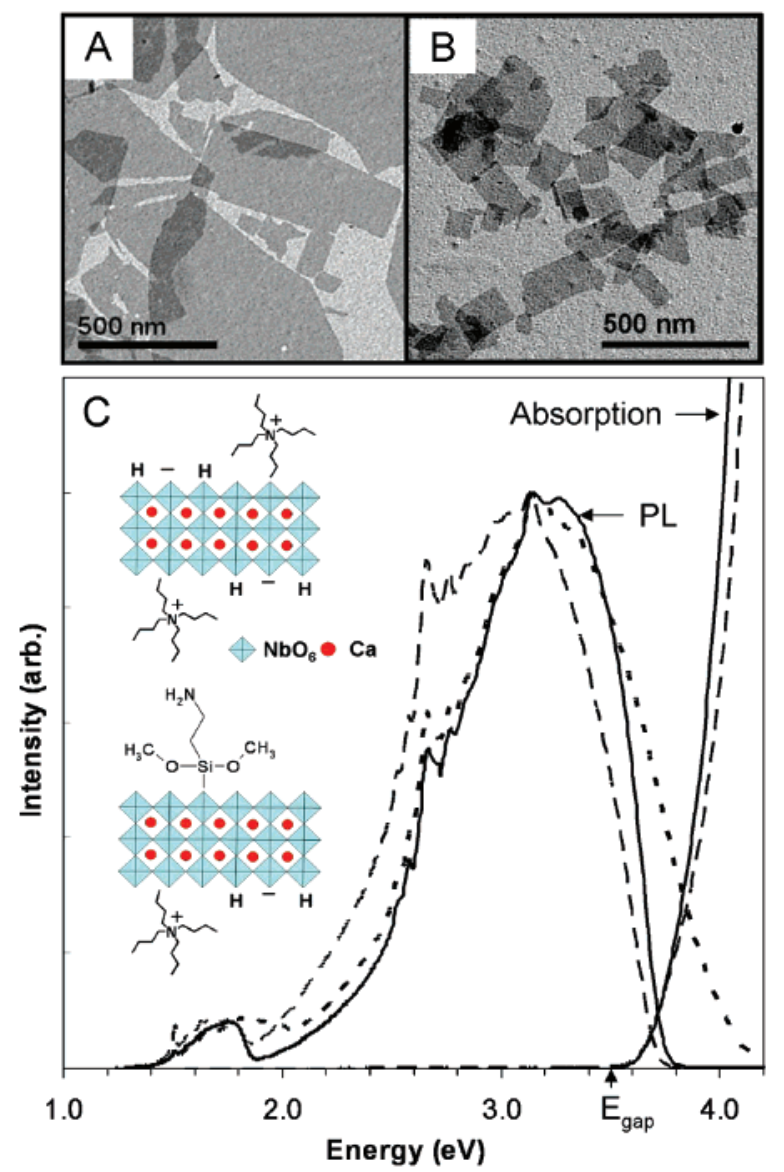

Figure 1. (A) TEM image of exfoliated $\mathrm{TBA}_{x}\left[\mathrm{H}_{1-x} \mathrm{Ca}_{2} \mathrm{Nb}_{3} \mathrm{O}_{10}\right](x=$ $0.15-0.20)$ nanosheets. (B) TEM image of $\mathrm{TBA}_{x}\left[\mathrm{H}_{1-x} \mathrm{Ca}_{2} \mathrm{Nb}_{3} \mathrm{O}_{10}\right]$ nanosheets after $30 \mathrm{~min}$ of ultrasonication. (C) Static UV-vis absorption and normalized PL spectra for dispersed $\mathrm{TBA}_{x}\left[\mathrm{H}_{1-x} \mathrm{Ca}_{2} \mathrm{Nb}_{3} \mathrm{O}_{10}\right]$ nanosheets with average edge lengths of $1.2 \pm 0.6 \mu \mathrm{m}$ (solid) and 80 $\pm 50 \mathrm{~nm}$ (dashed) and for (APS) $\mathrm{Ca}_{2} \mathrm{Nb}_{3} \mathrm{O}_{10}$ (dotted). The absorption spectra were corrected for Rayleigh scattering. Inset: schematic structure of $\mathrm{TBA}_{x}\left[\mathrm{H}_{1-x} \mathrm{Ca}_{2} \mathrm{Nb}_{3} \mathrm{O}_{10}\right]$ sheets with TBA counter-ions and of (APS) $\mathrm{Ca}_{2} \mathrm{Nb}_{3} \mathrm{O}_{10}$.

spectrum, and intensely sonicated nanosheets had considerably weaker scatter due to their reduced size. Correcting for a $\omega^{4}$ scattering component revealed that the nanosheets have an absorption edge at $3.53 \pm 0.05 \mathrm{eV}(\sim 350 \mathrm{~nm})$. This represents an increase in the band gap from both $\mathrm{KCa}_{2} \mathrm{Nb}_{3} \mathrm{O}_{10}\left(E_{\mathrm{g}}=3.35\right.$ $\mathrm{eV})^{1}$ and the parent bulk (TBA, $\left.\mathrm{H}\right) \mathrm{Ca}_{2} \mathrm{Nb}_{3} \mathrm{O}_{10}\left(E_{\mathrm{g}}=3.33 \mathrm{eV}\right)^{32}$ and indicates a relatively localized excited state in the exfoliated sheet. A two-dimensional quantum confinement argument is also supported by the sheet thickness $(1.16 \mathrm{~nm})$ and may be related to the reduced coordination of corner-shared $\mathrm{NbO}_{6}$ octahedra at the sheet surface. ${ }^{33}$ Comparable increases in the band gap have previously been observed between bulk and exfoliated (TBA, $\mathrm{H}) \mathrm{Ca}_{2} \mathrm{Nb}_{3} \mathrm{O}_{10}$ sheets prepared by an alternative method by Han et al. ${ }^{32}$ and in restacked sheets of a related perovskite, $\mathrm{K}_{4} \mathrm{Nb}_{6} \mathrm{O}_{17}$, by Furube et al. ${ }^{26}$ Near the threshold, the absorption coefficient was proportional to $\left(h v-E_{\mathrm{g}}\right)^{3}$, corresponding to an indirect band gap semiconductor. ${ }^{34}$

The photoluminescence (PL) spectrum in Figure 1 exhibited two broad peaks around $3.25 \mathrm{eV}(381 \mathrm{~nm})$ and $1.7 \mathrm{eV}(730$ $\mathrm{nm})$. The larger peak can be ascribed to band-edge emission, where a large Stokes shift is characteristic of a delocalized excited state in layered perovskite oxides. ${ }^{35}$ The PL from smaller sheets was red-shifted with a higher intensity in the low-energy emission. While this change may reflect an additional quantum size due to the smaller lateral dimensions, it is also likely that 

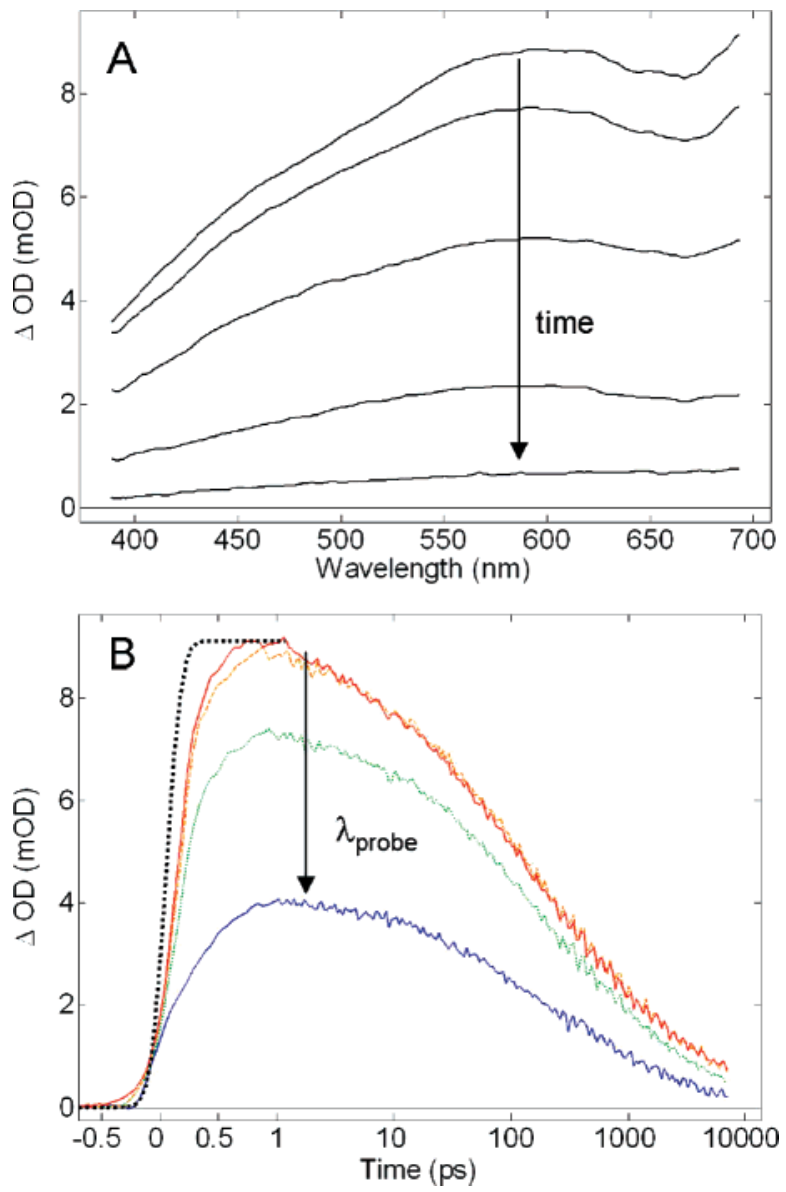

Figure 2. Transient absorption data of $\left[\mathrm{H}_{1-x} \mathrm{Ca}_{2} \mathrm{Nb}_{3} \mathrm{O}_{10}\right]^{x-}$ nanosheets in water at $\mathrm{pH}$ 10.5. (A) Transient spectra at $1 \mathrm{ps}, 10 \mathrm{ps}, 100 \mathrm{ps}, 1 \mathrm{~ns}$, and $7 \mathrm{~ns}$. (B) Kinetics at 400, 500, 600, and $690 \mathrm{~nm}$. A 240 fs Gaussian instrument response is shown for comparison. The pulse intensity was $0.7 \mathrm{~W} / \mathrm{cm}^{2}$.

the changes in emission arise from a higher density of surface defects in the smaller sheets. Radiative recombination involving trapped carriers would result in emission in the same spectral region as band-edge emission as well as extend to longer wavelengths. The longest wavelength emission peak probably originates from carriers deeply trapped in surface states resulting from lattice defects and dangling bonds at the sheet edge. The PL spectrum of smaller sheets was similar to PL observed in restacked (TBA, $\mathrm{H}) \mathrm{Ca}_{2} \mathrm{Nb}_{3} \mathrm{O}_{10}$ sheets, where an increase in defects was also expected. ${ }^{21}$ Nanosheets functionalized with 3-aminopropyl(trimethoxy)silane exhibited blue-shifted PL overlapping with the absorption edge. The PL intensity of the APS$\mathrm{Ca}_{2} \mathrm{Nb}_{3} \mathrm{O}_{10}$ sheets was about half the magnitude of nonfunctionalized sheets of a similar size, although intense scattering produced a large error on the absolute PL intensity, and quantum efficiencies were not determined from the data.

3.2. Transient Absorption in Water. The transient spectrum observed in dispersed (TBA, $\mathrm{H}) \mathrm{Ca}_{2} \mathrm{Nb}_{3} \mathrm{O}_{10}$ sheets in water consisted of a broad peak with a maximum around $600 \mathrm{~nm}$ (Figure 2A). This absorption feature has been observed in a wide range of metal oxide nanoparticles ${ }^{26,36-38}$ and is typically attributed to interband transitions of charge carriers trapped in mid-band states. Intraband transitions of free electrons are thought to generate an absorption spectrum that increases with wavelength to a mid-infrared peak following the conduction band density of states; intraband transitions contribute less to the visible transient absorption. ${ }^{28}$ Therefore, the rise of the transient absorption signals relates directly to the carrier trapping

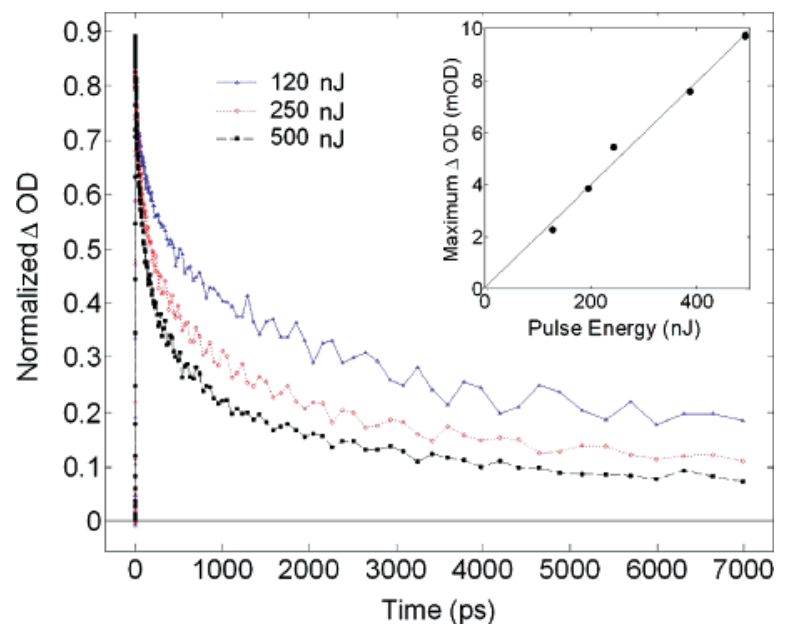

Figure 3. Transient decay slows with decreasing pump fluence $\left(\lambda_{\text {probe }}\right.$ $=650 \mathrm{~nm}$ ). Inset: initial transient amplitude increases linearly with pump fluence.

times (Figure 2B), which were not instrument-limited, indicating that carrier trapping occurs on a time scale greater than $\sim 200$ fs. Moreover, the time constant of the rise depended on wavelength and was distinctly slower in the blue than in the red spectral region. The transient absorption reached a maximum value for all wavelengths within $1 \mathrm{ps}$. The decay of the transient spectrum is related to the relaxation of carrier populations, which can occur through electron-hole recombination, non-radiative decay, or surface oxidation-reduction reactions. In water, the transient spectrum did not decay uniformly, with red wavelengths decaying slightly more quickly than blue wavelengths. Generally, spectral evolution indicates that multiple species contribute to the transient spectrum with different relaxation dynamics; however, photoinduced species can be difficult to distinguish if they have similar absorption spectra or if the dynamics of multiple species are coupled.

The intensity of the transient signal immediately after excitation scaled linearly with pump energy, indicating that linear excitation conditions applied. The observed carrier relaxation dynamics in water depended appreciably on laser fluence with the rate of decay slower with decreasing pulse energy; the trend is evident when the kinetic traces are normalized (Figure 3 ). The half-life $\tau_{1 / 2}$ of photogenerated trapped carriers was determined as the half-width-half-maximum (hwhm) of the transient decay. At all probe wavelengths, $\tau_{1 / 2}$ depended inversely on excitation fluence (Figure 4). This is consistent with second-order kinetic processes in which the rate of decay depends on the number of electron-hole pairs present in the nanosheet because the carriers can undergo non-geminate recombination. Approximately $10^{8}$ photons were absorbed per sheet in the highest fluence experiment due to the large particle size. Considering that the average nanosheet contained on the order of $10^{7} \mathrm{NbO}_{6}$ octahedra, the exciton density was comparable to studies of metal oxide nanoparticles conducted under similar experimental conditions ${ }^{39-41}$ and within the range where intensity-dependent kinetics would be expected. ${ }^{36}$

3.3. Kinetics Dependence on Nanosheet Size. Transient absorption measurements of intensely sonicated sheets revealed that the carrier decay dynamics depend on the size of the nanosheet (Figure 5). The smaller sheets exhibited an appreciably slower decay at all wavelengths, and was most pronounced at blue wavelengths. As shown in Figure 4, the transient half-life continued to follow the $I^{-1}$ trend of the larger sheets. The transient spectrum immediately following excitation has a slightly stronger absorption at blue wavelengths than in 


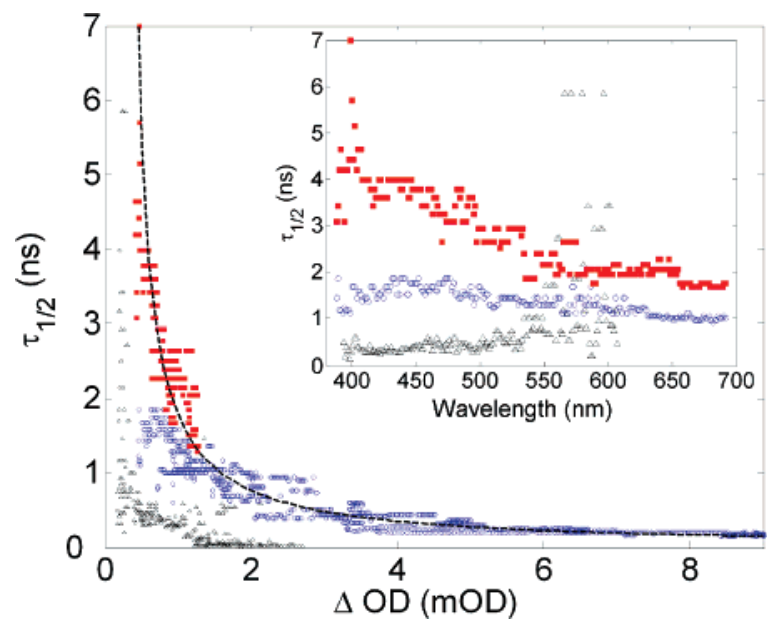

Figure 4. Half-life of the transient decay at all probe wavelengths in $\mathrm{TBA}_{x}\left[\mathrm{H}_{1-x} \mathrm{Ca}_{2} \mathrm{Nb}_{3} \mathrm{O}_{10}\right](x=0.15-0.20)$ sheets with an edge length of $1.2 \mu \mathrm{m}$ (blue circles) and $80 \mathrm{~nm}$ (red squares) depends inversely on signal magnitude, which was proportional to excitation fluence, $I$. The line $I^{-1}$ is shown for comparison. Surface-modified sheets (black triangles) exhibited faster relaxation. Inset: half-life decreases with wavelength, and the trend was stronger in the smaller sheets. Surfacemodified sheets exhibited an opposite trend, and the signal magnitude is nearly zero for $\lambda_{\text {probe }}>620 \mathrm{~nm}$.
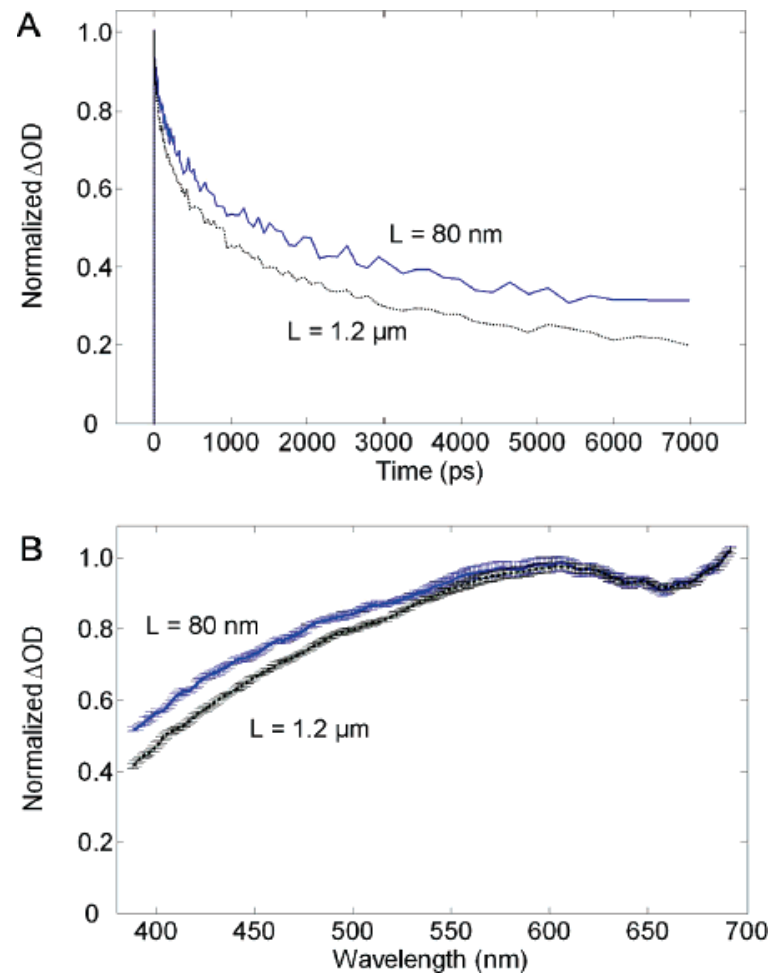

Figure 5. (A) Comparison of the transient decay $\left(\lambda_{\text {probe }}=650 \mathrm{~nm}\right)$ in $\mathrm{TBA}_{x}\left[\mathrm{H}_{1-x} \mathrm{Ca}_{2} \mathrm{Nb}_{3} \mathrm{O}_{10}\right](x=0.15-0.20)$ nanosheets of different average edge lengths, $L$. The excitation fluence for both samples is $0.4 \mathrm{~W} / \mathrm{cm}^{2}$. (B) Transient spectrum at $1.5 \mathrm{ps}$ shows that the smaller nanosheets absorb more strongly at blue wavelengths.

the larger sheets (Figure 5B). The initial transient spectrum did not depend significantly on the excitation fluence.

3.4. Surface Modification with 3-Aminopropyl(trimethoxy)silane. Because we are interested in using lamellar semiconductors such as $\left[\mathrm{H}_{1-x} \mathrm{Ca}_{2} \mathrm{Nb}_{3} \mathrm{O}_{10}\right]^{x-}$ as a building block for more complex photocatalyst nanostructures, we investigated the effect of a potential linking agent, 3-aminopropyl(trimethoxy)silane (APS). APS covalently bonds to the niobate surface through a $\mathrm{Si}-\mathrm{O}-\mathrm{Nb}$ linkage (Figure $1 \mathrm{C}$, inset). Previous studies have
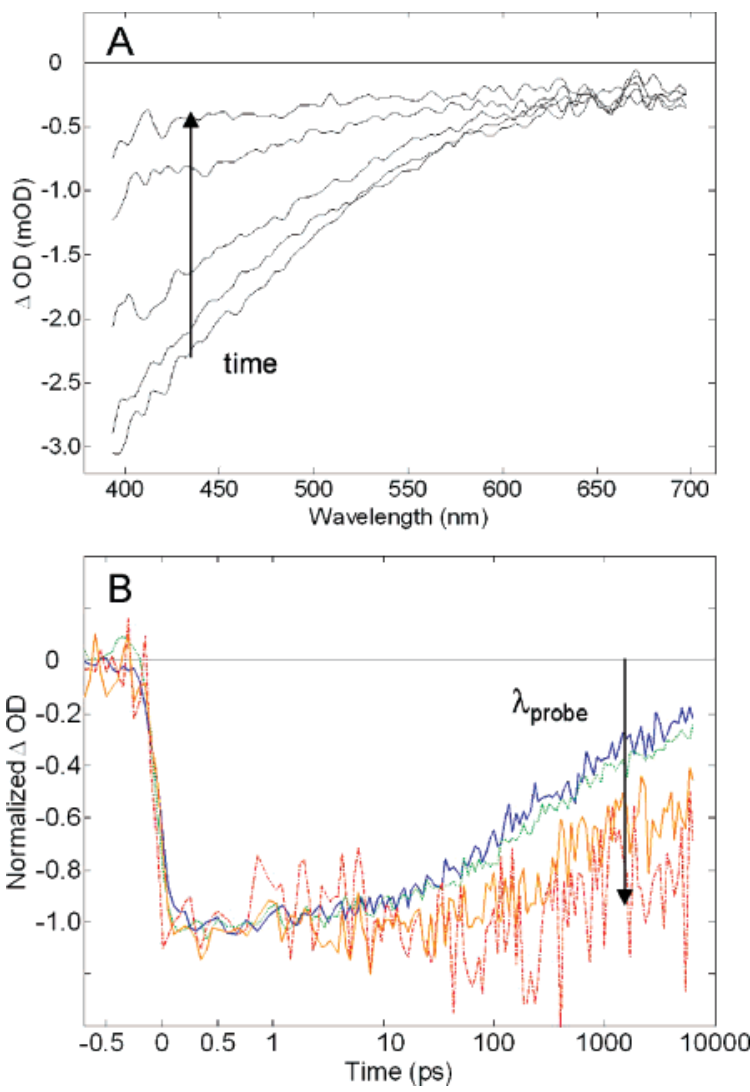

Figure 6. Transient absorption data of APS-functionalized $\mathrm{TBA}_{x}\left[\mathrm{H}_{1-x} \mathrm{Ca}_{2}\right.$ $\left.\mathrm{Nb}_{3} \mathrm{O}_{10}\right](x=0.15-0.20)$ sheets in water. (A) Transient spectra at 1 ps, $10 \mathrm{ps}, 100 \mathrm{ps}, 1 \mathrm{~ns}$, and $6.8 \mathrm{~ns}$. (B) Normalized kinetics at 400, 500,600 , and $695 \mathrm{~nm}$. The pulse fluence was $0.4 \mathrm{~W} / \mathrm{cm}^{2}$.

indicated that APS bonds to $21.5 \%$ of the surface $\mathrm{Nb}-\mathrm{O}$ sites, about half of the protonated sites on the nanosheets. ${ }^{23}$

As shown in Figure 6, the transient spectrum was significantly different in APS-functionalized niobate nanosheets than in nonfunctionalized samples. At all times and wavelengths, $\triangle \mathrm{OD}$ was negative, indicating that the transient spectrum was dominated by stimulated emission. The rise of this signal was instrumentlimited and displayed no wavelength dependence, but the rate of decay was faster for higher energy emission (Figure 6B). This pattern of spectral evolution is consistent with our interpretation of the photoluminescence spectrum as both bandedge and trapped-state emission, where radiative recombination corresponding to shallowly trapped or free carriers occurs relatively quickly, while recombination involving deeply trapped carriers occurs more slowly at longer wavelengths.

As with trapped carrier signals of unmodified niobiate sheets, the half-life of the stimulated gain depended on excitation fluence with an approximate $I^{-1}$ dependence (Figure 4). However, the decay kinetics was faster in functionalized sheets than in non-functionalized sheets for a comparable signal magnitude. This further suggests that the observed decay corresponds to the radiative recombination of shallowly trapped or free carriers.

3.5. Transient Absorption in Methanol. Methanol was added to a suspension of non-functionalized (TBA, $\mathrm{H}) \mathrm{Ca}_{2} \mathrm{Nb}_{3} \mathrm{O}_{10}$ nanosheets to act as a hole scavenger. Figure 7 shows the kinetics at 450 and $650 \mathrm{~nm}$ for nanosheet suspensions in 0,5 , 10,25 , and $50 \%$ (v) methanol. At all wavelengths, the rate of decay differed from water dynamics with as little as $5 \%$ methanol. The signals exhibited an initial increase in the decay rate with increasing methanol concentration, and a clear dependence on the probe wavelength was observed. The transient half-life was significantly reduced across all wavelengths 

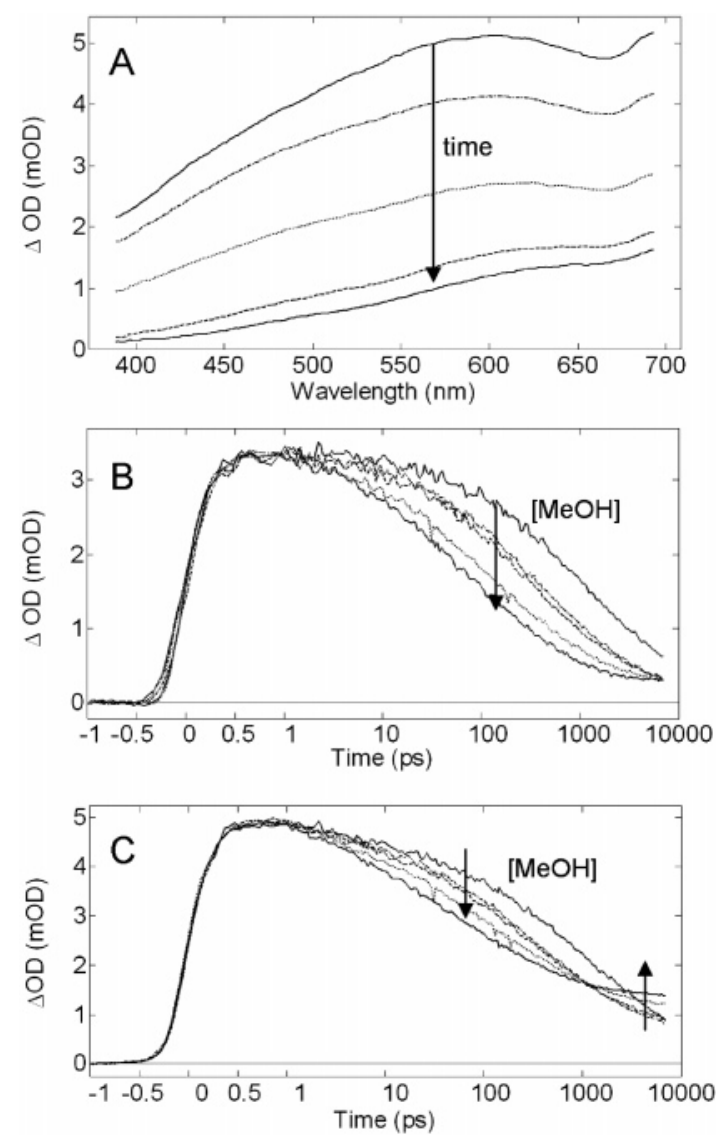

Figure 7. (A) Evolution of the transient spectrum of $\left[\mathrm{H}_{1-x} \mathrm{Ca}_{2} \mathrm{Nb}_{3} \mathrm{O}_{10}\right]^{x-}$ sheets suspended in a 1:1 methanol/water solution. (B) Kinetics in solutions of $0,5,10,25$, and $50 \%$ methanol at $450 \mathrm{~nm}$ show that the rate of relaxation increased with the addition of a hole scavenger. (C) Kinetics at $650 \mathrm{~nm}$ showed the formation of an absorption plateau.

in methanol. As the blue absorption decays to nearly zero, an absorption plateau was established at red wavelengths within a few nanoseconds. There was no noticeable change in the shape or magnitude of the absorption spectrum between $2 \mathrm{~ns}$ and the latest time probed ( $7 \mathrm{~ns})$. If the sample volume was not refreshed at the $1-\mathrm{kHz}$ repetition rate of the laser, we observed a pretime zero signal that indicated that the transient absorption spectrum did not change over $1 \mathrm{~ms}$. The magnitude of the transient signal at $7 \mathrm{~ns}$ increased with methanol concentration.

The spectral evolution in aqueous methanol solutions demonstrates that the transient signal consisted of at least two contributing species, which we will identify as trapped electrons and trapped holes. Whereas in water, the electron and hole dynamics were likely coupled through recombination (and therefore difficult to distinguish by relatively weak spectral evolution), methanol decoupled the relaxation dynamics through photocatalytic redox reactions. Adsorbed methanol reduces holes at the sheet surface, leaving the nanosheet with an excess of photogenerated electrons. The reduced half-life and the observation that the red-shifted transient absorption spectrum was stable on a millisecond time scale (from a pre-time zero signal) both indicate that photo-oxidation of methanol occurs within a few nanoseconds. We assigned the long-lived transient spectrum in $50 \%$ methanol to trapped electrons, and by assuming that electrons are the only species contributing at $690 \mathrm{~nm}$, the spectrum of the trapped hole was calculated as the remainder of the transient spectrum in water. The resulting hole spectrum $\alpha_{\mathrm{h}}(\lambda)$ had a broad peak around $470 \mathrm{~nm}$, as shown in Figure 8, and was found to be insensitive to pump fluence and methanol concentration $(0-50 \%)$. Error bars on the recovered spectrum in Figure 8A represent the standard deviation among all the data sets with larger sheets. Significant deviation from $\alpha_{h}(\lambda)$ was observed in smaller sheets as a result of the aforementioned differences in the transient spectrum (Figure 5B).

To characterize the dynamics of both trapped carrier populations, each measured transient spectrum was decomposed into separate population functions $C_{\mathrm{e}}(t)$ and $C_{\mathrm{h}}(t)$

$$
\Delta \mathrm{OD}(t, \lambda)=C_{\mathrm{e}}(t) \alpha_{\mathrm{e}}(\lambda)+C_{\mathrm{h}}(t) \alpha_{\mathrm{h}}(\lambda)
$$

The relative extinction coefficients $\alpha_{\mathrm{e}}$ and $\alpha_{\mathrm{h}}$ were decided under an assumption of equal concentrations of trapped electrons and trapped holes at long times ( $7 \mathrm{~ns})$ in water. With the exception of the intensely sonicated sheet samples, the transient data decomposed into the same two species with less than 5\% error at all time points. The resulting evolution of carrier populations in water and in 50\% methanol is shown in Figure 8. Notably, the trapped electron and trapped hole $\left(C_{\mathrm{e}}(t)\right.$ and $\left.C_{\mathrm{h}}(t)\right)$ relaxation dynamics differed even in water. The slower rise of the blue edge of the transient spectrum was seen to correspond to the generation of trapped holes, while electrons were trapped more quickly. However, the hole trapping time decreased in the presence of methanol. Both carrier populations decayed on multiple time scales.

3.6. Analysis of Carrier Dynamics. Extracting microscopic carrier relaxation rates in nanocrystalline semiconductors is a challenging and well-studied problem. ${ }^{36,40-44}$ While nongeminate electron-hole recombination has the character of a bimolecular reaction, second-order kinetics slows down until, at a sufficiently low carrier concentration, the limited number of electron-hole pairs on an individual nanosheet cannot satisfy the statistical assumptions implicit in conventional rate laws. ${ }^{36,43}$ Geminate carrier recombination under low carrier concentration is thought to be limited by trapped carrier diffusion, and when diffusion is confined to two dimensions (e.g., by the lamellar sheet geometry), the second-order recombination rate asymptotically approaches a $t^{-1 / 2}$ dependence according to theory. ${ }^{43}$

Several complications arise in determining relaxation rates due to the material properties of the (TBA, $\mathrm{H}) \mathrm{Ca}_{2} \mathrm{Nb}_{3} \mathrm{O}_{10}$ nanosheet samples: Low-resolution TEM images indicated that the nanosheets are polydispersed. Despite an expected Poissonian distribution of photogenerated electron-hole pairs, ${ }^{36,43}$ larger sheets will on average have higher initial carrier concentrations, and this could affect the recombination dynamics if the carriers were delocalized or mobile enough to traverse a significant area of the sheet to interact. Thus, the size inhomogeneity potentially contributes considerable uncertainty to the ensemble spectroscopic measurement. Furthermore, high-resolution TEM studies found that unilamellar niobate nanosheets prepared by the same methods as we have used can vary in (non)stoichiometric composition and surface structure ${ }^{45}$ which can be expected to affect excited-state characteristics. Incomplete knowledge of the inhomogeneous distribution significantly limits the utility of mechanistic rate equation models.

We chose then to derive relevant relaxation time scales from a phenomenological fit of the kinetic data. Each carrier population profile extracted from the decomposition described previously was fit with a series of second-order decays, representing two sub-populations with a common trapping time constant

$$
\begin{array}{r}
C(t)=G(t) \otimes \exp \left(-k_{\text {trap }} t\right) \otimes\left[C_{0}\left(1+C_{0} k_{1}{ }^{\prime} t\right)^{-1}+\right. \\
\\
\left.C_{1}\left(1+C_{1} k_{2}{ }^{\prime} t\right)^{-1}\right]
\end{array}
$$

where $G(t)$ is a Gaussian instrument response function, $k^{\prime}=$ 

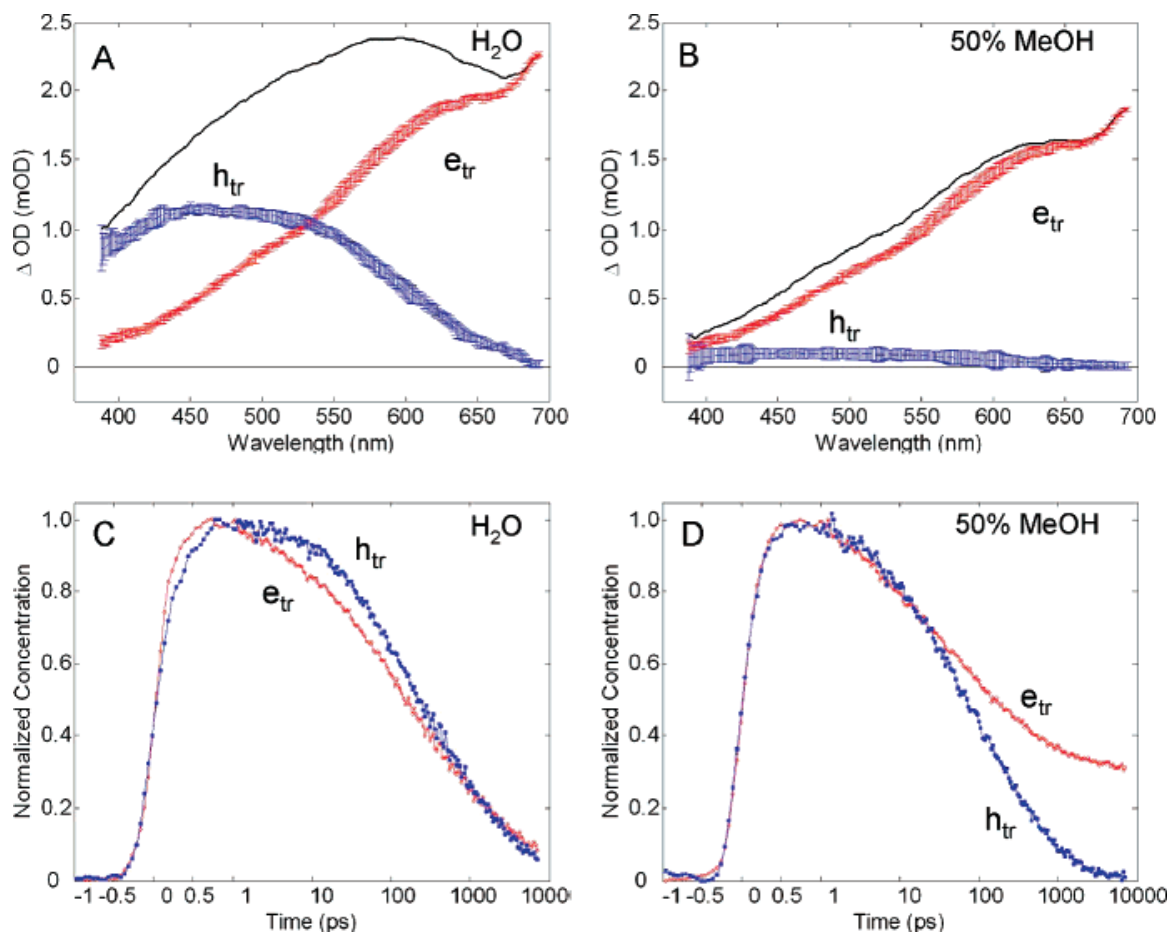

Figure 8. (A) Decomposition of the transient spectrum at $1 \mathrm{~ns}$ into trapped hole and trapped electron populations in water and (B) 1:1 water-/ methanol solution at $\mathrm{pH} \cong 10.5$. (C) Normalized extracted concentration profiles of the trapped carrier electrons in water and (D) $1: 1$ water-/ methanol solution. The pulse fluence was $0.7 \mathrm{~W} / \mathrm{cm}^{2}$ in water and $0.5 \mathrm{~W} / \mathrm{cm}^{2}$ in methanol.
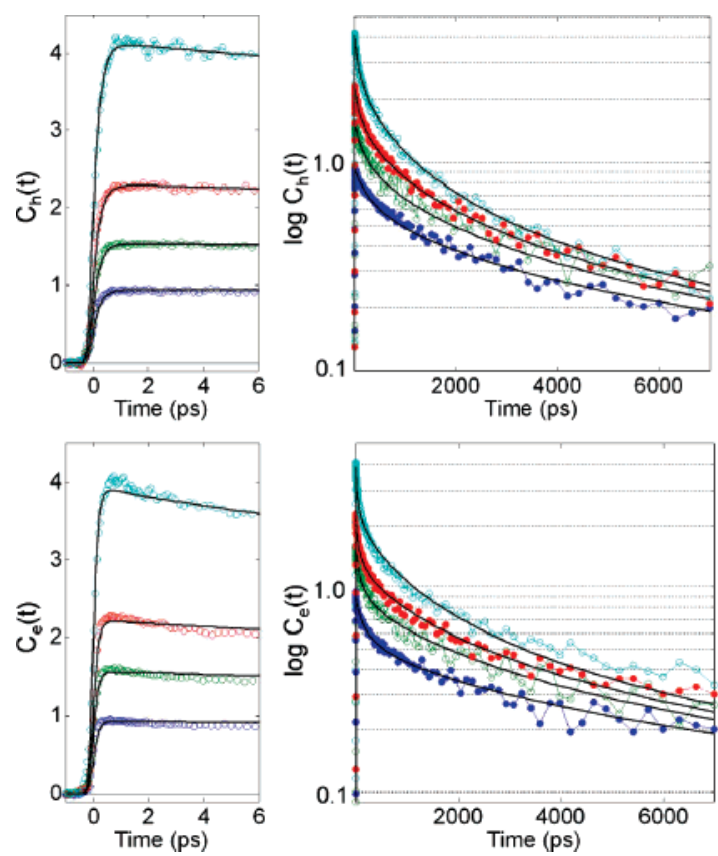

Figure 9. Hole (top) and electron (bottom) profiles extracted from $\left[\mathrm{H}_{1-x} \mathrm{Ca}_{2} \mathrm{Nb}_{3} \mathrm{O}_{10}\right]^{x-}$ sheets in water at $\mathrm{pH} 10.5$ at four excitation energies. Multicomponent fits are shown with solid curves. Parameters are found in Table 1.

$k_{i} / \alpha(i=1,2)$, and $\alpha$ is an extinction coefficient. The coefficient $\alpha$ and the rate constants $k_{\text {trap }}, k_{1}$, and $k_{2}$ were constrained to simultaneously fit data at five excitation fluences while the initial sub-populations $C_{0}$ and $C_{1}$ were taken to be proportional to laser fluence. In the trapped holes, the ratio $C_{0} / C_{1}$ was found to be approximately 1 , and the fit was not appreciably worsened under the constraint $C_{1}=C_{0}$. Extracted carrier traces and fits from four excitation fluences are shown in Figure 9, and the fitting parameters obtained from eq 2 for electron and hole populations are summarized in Table 1 . The model fit to $C_{\mathrm{h}}(t)$ was
TABLE 1: Second-Order Time Constants Obtained in $\left[\mathrm{H}_{1-x} \mathrm{Ca}_{2} \mathrm{Nb}_{3} \mathrm{O}_{10}\right]^{x-}$ Nanosheets in Water ${ }^{a}$

\begin{tabular}{cllll}
\hline av edge length & carrier & $k_{\text {trap }}{ }^{-} 1(\mathrm{ps})$ & ${k_{1}{ }^{-} 1(\mathrm{ps})}$ & $k_{2}{ }^{-} 1(\mathrm{ps})$ \\
\hline $1.2 \pm 0.6 \mu \mathrm{m}$ & electron & $0.13(0.02)$ & $8(2)$ & $280(20)$ \\
$1.2 \pm 0.6 \mu \mathrm{m}$ & hole & $0.24(0.01)$ & $19(2)$ & $260(20)$ \\
$80 \pm 50 \mathrm{~nm}$ & electron & $0.18(0.02)$ & $10(2)$ & $430(50)$ \\
$80 \pm 50 \mathrm{~nm}$ & hole & $0.22(0.01)$ & $20(4)$ & $520(20)$
\end{tabular}

${ }^{a}$ Standard deviations are shown in parentheses.

considerably better than to $C_{\mathrm{e}}(t)$ (Figure 9), indicating that the trapped electron dynamics was more complicated than the trapped hole dynamics.

Populations derived from the sample of smaller sheets were also fit to eq 2 and exhibited significantly slower relaxation, as suggested earlier by the wavelength-resolved transient half-lives. To obtain equal concentrations of trapped carriers on a nanosecond time scale, it was necessary to assign a larger extinction coefficient to the trapped hole than previously determined in the larger sheets because of the higher relative absorption of blue in the smaller sheets. The resulting trapped electron concentration is initially larger than the trapped hole concentration (Supporting Information), in contrast to the larger sheets. The reconstructed data also had significant residuals below $430 \mathrm{~nm}$ exhibiting the same dynamics as the trapped hole, which suggested that the actual spectrum of the trapped hole in smaller sheets is blue-shifted as compared to in larger sheets. Photocatalytic measurements have shown that any increase in $\mathrm{H}_{2}$ evolution for smaller sheets is within experimental error (data not shown).

Independent fits of $C_{\mathrm{e}}(t)$ and $C_{\mathrm{h}}(t)$ did not explicitly couple the dynamics of trapped holes and electrons, although the assumption of equal final carrier concentration in water fixed the extinction coefficients $\alpha_{h}$ and $\alpha_{e}$ to be of comparable magnitude. Nonetheless, the similarity in the slowest rate constants and second-order nature of the nanosecond kinetics suggests that trapped electron-trapped hole recombination was the primary relaxation mechanism on the sub-nanosecond time scale. 

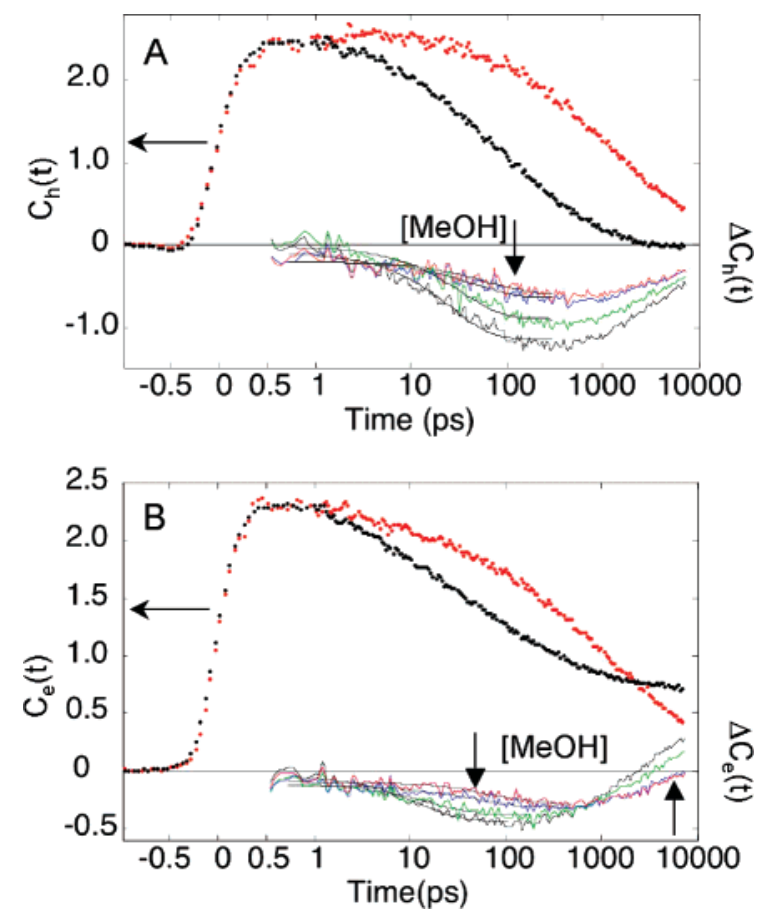

Figure 10. (A) Difference in trapped electron in water and methanol/ water solution. (B) Difference in trapped hole concentration in water and methanol/water solution. The early component of each population difference was fit to a single-exponential decay (Table 2).

In methanol, the dynamics of the carriers were correlated both through recombination and through surface reactions. The photocatalytic surface reactions were analyzed by plotting the differences between trapped carrier populations derived from niobate nanosheets in water and in each aqueous methanol solution, as shown in Figure 10. The population differences $\Delta C_{\mathrm{e}}(t)$ and $\Delta C_{\mathrm{h}}(t)$ indicated that both $C_{\mathrm{e}}(t)$ and $C_{\mathrm{h}}(t)$ were diminished in methanol for the first nanosecond following photoexcitation. The persistent decrease in $C_{\mathrm{h}}(t \gg 0)$ is consistent with an additional relaxation path for trapped holes through the photo-oxidation of methanol. The initially diminished electron population in methanol also suggests that enhanced photo-oxidation of the niobate nanosheet occurred.

Both $\Delta C_{\mathrm{e}}(t)$ and $\Delta C_{\mathrm{h}}(t)$ reached a turning point $(\mathrm{d} \Delta C(t) / \mathrm{d} t$ $=0$ ) when the combined rate of decay due to surface redox chemistry and electron-hole recombination was equal to the rate of recombination in water. Following this point, both $C_{\mathrm{e}}(t)$ and $C_{\mathrm{h}}(t)$ had slower relaxation dynamics in methanol than in water. The slowing of recombination dynamics is due to the imbalance of carrier populations and can be interpreted as the competition between surface redox reactions and electron-hole recombination. Once $C_{\mathrm{h}}(t)$ was fully depleted, the electron population $C_{\mathrm{e}}(t)$ in methanol $(>25 \%)$ surpassed the final measured electron population in water.

The sub-nanosecond components of $\Delta C_{\mathrm{e}}(t)$ and $\Delta C_{\mathrm{h}}(t)$ were each fit to an exponential decay, and the time constants are presented in Table 2 . The rate constants increased linearly with methanol concentration. This suggests that adsorption was not the rate limiting step in the oxidation/reduction reaction and that the surface reaction likely involved adsorbed methanol molecules.

\section{Discussion}

4.1. Trapped Carrier Spectra. While the transient spectra in water contained weakly resolvable electron-hole dynamics, the interaction of a hole scavenger left the semiconductor
TABLE 2: Exponential Fit Parameters for Difference in Trapped Carrier Populations Derived from $(\mathrm{TBA}, \mathrm{H}) \mathrm{Ca}_{2} \mathrm{Nb}_{3} \mathrm{O}_{10}$ Nanosheets in Water and Methanol Solutions ${ }^{a}$

\begin{tabular}{ccc}
\hline methanol \% & $k_{\mathrm{ox}}{ }^{-} 1(\mathrm{ps})$ & $k_{\mathrm{re}}{ }^{-1}(\mathrm{ps})$ \\
\hline 5 & $60(20)$ & $76(15)$ \\
10 & $45(15)$ & $48(15)$ \\
25 & $33(5)$ & $23(3)$ \\
50 & $25(5)$ & $12(2)$ \\
&
\end{tabular}

nanosheet with an excess of photogenerated electrons. Therefore, with the introduction of methanol, we separated the electron spectrum with a shoulder at $690 \mathrm{~nm}$ from the trapped hole spectrum with a broad peak around $470 \mathrm{~nm}$. Similar absorption spectra for photogenerated electrons have been identified in nanocrystalline $\mathrm{TiO}_{2},{ }^{46}$ where the spectrum of the trapped electron overlapped the free electron absorption spectrum but was distinguished by an absorption peak around $770 \mathrm{~nm}$ due to localization. Here, we attributed the higher energy shoulder around $690 \mathrm{~nm}$ to electrons in deep trap states.

The origin of the trapped hole spectrum is generally less clear but is known to be sensitive to the nature of the trap state. ${ }^{37,46}$ Recently, Yoshihara et al. identified two distinct trapped hole spectra in nanocrystalline $\mathrm{TiO}_{2}$ that appeared in different concentrations depending on the solution $\mathrm{pH} .{ }^{47}$ They suggested that one absorption spectrum (which had a peak $<400 \mathrm{~nm}$ ) arose from charge transfer complexes between shallowly trapped holes and hydroxide ions because the spectrum disappeared in an acidic solution. The trapped hole spectrum in $\left[\mathrm{H}_{1-x} \mathrm{Ca}_{2} \mathrm{Nb}_{3} \mathrm{O}_{10}\right]^{x-}$ may similarly originate from a surface complex. If holes were locally trapped on electron-rich surface oxygen on the $\left[\mathrm{H}_{1-x} \mathrm{Ca}_{2} \mathrm{Nb}_{3} \mathrm{O}_{10}\right]^{x-}$ sheet, this interpretation would explain the absence of trapped hole absorption in the APS surface-modified niobate sheets. Yoshihara et al. ${ }^{46}$ also proposed that a visible trapped hole spectrum could originate from an energy splitting of surface dimers caused by lattice deformations. In this case, the nature of the spectrum in $\left[\mathrm{H}_{1-x} \mathrm{Ca}_{2} \mathrm{Nb}_{3} \mathrm{O}_{10}\right]^{x-}$ (which is slightly higher in energy than in $\mathrm{TiO}_{2}$ ) has greater implications about the niobate valence band structure and bandwidth. This question may be elucidated by detailed calculation of the valence band structure in (TBA, $\mathrm{H}) \mathrm{Ca}_{2} \mathrm{Nb}_{3} \mathrm{O}_{10}$ and identification of the exact nature of trapped hole states.

We found evidence that the trapped hole spectrum depends weakly on the size of the niobate nanosheet. The transient spectrum of smaller nanosheets had a greater absorption on the blue edge, particularly below $430 \mathrm{~nm}$ (Figure 5B), and the higher energy spectrum suggested a less localized or shallowly trapped valence band hole. We interpreted these differences as the trapped hole having a larger absorption coefficient, which would be expected from a shallow trap. ${ }^{47}$ The red-shift of the photoluminescence spectrum (Figure 1C) also indicated an increase in the density of the surface defects. The polydispersed samples of larger sheets may also contain some 80-nm sheets, in which case both trapped hole spectra should be present, but the spectra are probably too similar to distinguish inhomogeneous sub-populations of trapped carriers. The variation in the trapped hole spectrum may be studied in more detail with the aid of an electron scavenging agent. ${ }^{48}$

4.2. Trapping and Recombination Dynamics. A key motivation for identifying the trapped carrier spectra in $\left[\mathrm{H}_{1-x} \mathrm{Ca}_{2} \mathrm{Nb}_{3} \mathrm{O}_{10}\right]^{x-}$ nanosheets was to separately analyze electron and hole dynamics in photocatalytic nanostructures based on this material. The extracted populations in Figures $8-10$ indicate that trapped electrons and trapped holes experience 
different relaxation dynamics even in the absence of a scavenging agent. This discrepancy between the photodynamics of carriers has also been observed in $\mathrm{TiO}_{2}{ }^{28}$ and can arise from the carriers undergoing relaxation processes other than electronhole recombination. The phenomenological model in eq 2 adequately describes the dynamics of the species identified as the surface trapped hole, where the model can be interpreted as two independent sub-populations that decay on a 24-ps time scale and a 260-ps time scale. Multiple populations may either arise from inhomogeneity in the ensemble of nanosheets (predominately as sheets of different dimensions have an average number of electron-hole pairs per sheet) or from an energetic distribution of trap states within a nanosheet. There is not sufficient control over the sheet size at present to distinguish these two possibilities, and indeed, they may coexist. In either case, the model of eq 2 serves mainly to characterize the time scales on which trapped hole and electron dynamics occurs in water.

The photodynamics of trapped electrons was more complicated than the photodynamics of trapped holes. In particular, the initial decay component is not a single second-order decay and possibly represents additional trapped electron populations, thermal detrapping from shallow electron traps, or interaction between trap states not included in eq 2 . Tamaki et al. attributed a 500-ps exponential decay to equilibration between surface trapped electrons and shallowly trapped bulk electrons. ${ }^{28} \mathrm{We}$ expect that the majority of electrons in the $\left[\mathrm{H}_{1-x} \mathrm{Ca}_{2} \mathrm{Nb}_{3} \mathrm{O}_{10}\right]^{x-}$ nanosheets is associated with surface states due to the 1.16-nm sheet thickness and that an equilibration process more likely occurs between surface trap states of different energies. This process must be relatively fast to contribute to the observed relaxation dynamics in the presence of non-geminate recombination. Higher order electron processes such as Auger recombination are also possible at high carrier concentrations ${ }^{41}$ and, if present, could affect the earliest dynamics.

We ascribed the $\sim 270$-ps time scale found in both carriers to the recombination of trapped carriers. This time constant is nearly twice as long as the relaxation constant reported in an ultrafast study of restacked $\mathrm{K}_{4} \mathrm{Nb}_{6} \mathrm{O}_{17}{ }^{26}$ It is difficult, however, to compare literature values because the rate constants associated with second-order decay depend on the initial carrier concentration and vary with experimental conditions. Tamaki et al. ${ }^{28}$ successfully avoided the problem of second-order recombination dynamics with weak excitation fluence of nanocrystalline $\mathrm{TiO}_{2}$ where an average of electron-hole pairs is generated per particle and undergoes geminate recombination. On the relatively short time scale of that study $(1.5 \mathrm{~ns})$, no relaxation of holes was observed, and the relaxation of electrons was attributed to changes in the electron absorption cross-section rather than electron-hole recombination. Previous microsecond timeresolved studies of electron-hole recombination in $\mathrm{TiO}_{2}$ in the presence of a small number of excitations have found that nanosecond-microsecond transients follow a single-exponential decay. ${ }^{28,36}$ We expect that similar dynamics would be observed in $\left[\mathrm{H}_{1-x} \mathrm{Ca}_{2} \mathrm{Nb}_{3} \mathrm{O}_{10}\right]^{x-}$ nanosheets on a longer time scale when the transient relaxation reflects geminate recombination of deeply trapped carriers.

The recombination dynamics in smaller nanosheets was distinctly slower according both to the transient half-lives and the direct fits of electron and hole populations. Because the carriers undergo second-order recombination, slower rates can be expected from the smaller number of excitations per sheet. The excitation density was determined by the laser fluence, and smaller sheets will have, on average, fewer electron-hole pairs per sheet. In contrast, in nanocrystalline $\mathrm{TiO}_{2}$, recombination rates increase as the particle size decreases, and rapid surface recombination becomes the dominant relaxation process. ${ }^{44,49}$ However, this interpretation does not fully explain the slower hole dynamics, and differences in the relaxation dynamics may arise from other physical characteristics that depend on nanosheet size. Our interpretation of the trapped hole spectrum suggested that the concentration of trapped electrons was initially larger than the trapped hole concentration. One explanation for an increase in trapped electron density is the modest increase in sheet edges to surface area. The sheet edges contain dangling $\mathrm{Nb}-\mathrm{O}$ bonds that act as electron trap sites. We previously found that platinum nanoparticles preferentially attached to the edge of the nanosheets during photodeposition because of electron accumulation in this region. ${ }^{21}$

Distinct carrier trapping times were determined from the exponential rise of $C_{\mathrm{e}}(t)$ and $C_{\mathrm{h}}(t)$. The trapping of electrons and trapping of holes are largely independent processes that depend on the density of the trap states and the effective mass of the carrier. Trapped electrons developed within the instrument response function (exponential rise of $140 \mathrm{fs}$ ). The slower rise observed at high excitation fluence is possibly a trap filling effect, where the increasing Fermi energy of the photoexcited niobate sheet reduces the trap density. ${ }^{42}$ The trapping time observed for holes (220 fs) is relatively slow. Most hole trapping times reported in the literature are either instrument-limited or obscured by two-photon absorption, ${ }^{27,28}$ and several experiments with higher time resolutions did not study the absorption of the trapped hole. ${ }^{50}$ The slower trapping time for holes could originate from a larger effective mass or a smaller mobility. ${ }^{37,41}$

4.3. Photo-oxidation of Methanol. Both trapped holes and trapped electrons had distinctly faster dynamics in methanol than in water, indicating that efficient surface redox reactions occurred. The time scale for methanol photo-oxidation was determined to be $<100 \mathrm{ps}$ by analyzing the difference in the trapped hole populations derived from niobate nanosheets in methanol/water solutions and pure water. In a similar analysis, Tamaki et al. ${ }^{27,28}$ recently determined a 300-ps exponential time constant for photocatalytic oxidation of alcohols by $\mathrm{TiO}_{2}$ nanoparticles. The faster oxidation rates observed in $\left[\mathrm{H}_{1-x} \mathrm{Ca}_{2}\right.$ $\left.\mathrm{Nb}_{3} \mathrm{O}_{10}\right]^{x-}$ are consistent with photoelectrochemical experiments by Izawa et al., which determined that restacked $\left[\mathrm{H}_{1-x} \mathrm{Ca}_{2}\right.$ $\left.\mathrm{Nb}_{3} \mathrm{O}_{10}\right]^{x-}$ nanosheets have a much higher photocatalytic activity for methanol oxidation than $\mathrm{Ti}_{0.91} \mathrm{O}_{2}$ nanosheets (more than 6 times the photocurrent). ${ }^{29}$ The overlap of time scales showed competition between photo-oxidation and electron-hole recombination. Inhibited recombination was clear from the final amplitude of long-lived electrons that increased with the methanol concentration. Similarly, Colombo and Bowman observed picosecond competition between carrier recombination in $\mathrm{TiO}_{2}$ nanoparticles and the oxidation of the hole scavenger $\mathrm{SCN}^{-} .51$ This competition was not apparent in the Tamaki et al. study ${ }^{27,28}$ because the electron-hole recombination dynamics occurred on a longer time scale than photo-oxidation under their weak excitation conditions. Our results indicate that reduced surface recombination is responsible for a long-lived charge separation, in agreement with a long-lived charge separation observed in clay-niobate materials under steady-state illumination. ${ }^{52}$

We also observed a sub-nanosecond loss of electrons in $\left[\mathrm{H}_{1-x} \mathrm{Ca}_{2} \mathrm{Nb}_{3} \mathrm{O}_{10}\right]^{x-}$ that was linearly dependent on the methanol concentration. Methanol is also known to improve the photocatalytic efficiency of $\mathrm{H}_{2}$ generation from $\mathrm{H}_{2} \mathrm{O}$ on niobate sheets, ${ }^{29}$ although this is likely a result of the diminished surface 
recombination and excess of trapped electrons on a nanosecondmicrosecond time scale. Protonated sites on the niobate or adsorbed $\mathrm{H}_{2} \mathrm{O}$ may be reduced on the same time scale that methanol is first oxidized (before $100 \mathrm{ps)} \mathrm{because} \mathrm{there} \mathrm{is}$ already an excess of electrons. However, at 50\% methanol, the loss of electrons is faster than the loss of holes, and another reduction reaction may be involved. One possibility is the photoreduction of physisorbed methanol by a conduction band or shallowly trapped electrons. Niobate sheets have a higher energy conduction band potential than $\mathrm{TiO}_{2},{ }^{29}$ which could account for methanol reduction by trapped electrons in $\left[\mathrm{H}_{1-x} \mathrm{Ca}_{2} \mathrm{Nb}_{3} \mathrm{O}_{10}\right]^{x-}$, whereas Tamaki et al. found that bulk and surface trapped electrons in $\mathrm{TiO}_{2}$ were not affected by methanol. ${ }^{28,53}$ Photocatalysis and EPR studies are currently in progress to identify the process responsible for the loss of electrons.

A point of concern in these measurements is that the repetition rate of the laser $(1 \mathrm{kHz})$ is high enough to potentially observe an accumulation of electrons in the niobate sheet due to hole scavenging, either because the excitation volume was not refreshed at a sufficient rate or because scavenged sheets circulating through the reservoir were re-excited before they were reduced by the aqueous methanol solution. Re-excitation of a sheet with an excess of free or trapped electrons could affect carrier dynamics in several ways, including faster electron thermalization as a result of additional electron-electron scattering or an increased rate of non-geminate electron-hole recombination. To test for the possibility of re-exciting scavenged niobate sheets, we reduced the repetition rate of the laser to $500 \mathrm{~Hz}$. The observed dynamics was within the experimental error of the dynamics obtained using a $1-\mathrm{kHz}$ repetition rate. We conclude that the re-excitation of scavenged sheets does not contribute significantly to the observed dynamics and that electron accumulation on hole scavenged $\left[\mathrm{H}_{1-x} \mathrm{Ca}_{2} \mathrm{Nb}_{3} \mathrm{O}_{10}\right]^{x-}$ nanosheets negligibly contributes to the reported redox and recombination rates.

4.4. Surface-Modified Nanosheets. Chemical surface modification is particularly important in photocatalysts because the catalytic activity is sensitive to surface recombination and interfacial charge transfer. The absence of trapped carrier absorption in the APS-functionalized nanosheets suggests that the carrier dynamics was considerably altered as a result of changes in the surface composition. In particular, the lack of trapped hole absorption underlying the emission suggested a significant loss of hole trap sites. Because APS displaces approximately half of the protonated oxygen sites, ${ }^{23}$ it is possible that trapped holes are associated with the $\mathrm{H}-\mathrm{O}-\mathrm{Nb}$ bond, which would have a higher surface charge density than the $\mathrm{Si}-\mathrm{O}-$ $\mathrm{Nb}$ linkage. A reduced number of trapped holes was also consistent with the lower photoluminescence yield for the APSmodified material. Surface passivation can virtually eliminate $\mathrm{PL}^{54}$ as a result of the elimination of trapped hole states, as has been demonstrated in nanocrystalline chalcogenides. ${ }^{55-58}$

\section{Conclusion}

In this study, we characterized charge trapping and recombination dynamics in exfoliated $\left[\mathrm{H}_{1-x} \mathrm{Ca}_{2} \mathrm{Nb}_{3} \mathrm{O}_{10}\right]^{x-}$ nanosheets. Absorption spectra for trapped holes and trapped electrons were identified by the action of the methanol and subsequently were used to separate the electron and hole dynamics in water. The dynamics of trapped electrons and trapped holes were found to be distinct and was well-described using a phenomenological model involving two populations following second-order kinetics. Although this model overlooks many of the subtleties of charge trapping and relaxation processes, it provided an estimate of the charge separation time scales. The recombination of surface-trapped photogenerated electrons and holes primarily occurred on a sub-nanosecond time scale comparable to relaxation in other metal oxide nanocrystals. Surface modification with 3-aminopropyl(trimethoxy)silane resulted in the loss of surface trap sites, and faster band-edge radiative recombination occurred. Ultrafast interfacial charge transfer is therefore an important criterion in selecting linker ligands to make use of lamellar semiconductors such as $\left[\mathrm{H}_{1-x} \mathrm{Ca}_{2} \mathrm{Nb}_{3} \mathrm{O}_{10}\right]^{x-}$ as building blocks for more complex photocatalyst nanostructures.

We also directly observed the photo-oxidation of methanol on exfoliated niobate nanosheets and obtained a sub-100-ps time constant associated with photo-oxidation from the difference between methanol and water. Additional analysis of photooxidation dynamics and its relation to photocatalytic activity toward $\mathrm{H}_{2}$ generation in water will be presented in a future publication.

Acknowledgment. This work was supported by an Energy Innovation Startup Grant of the California Energy Commission and funds from the University of California.

Supporting Information Available: Additional analysis of the evolution of carrier populations in intensely sonicated $\left[\mathrm{H}_{1-x} \mathrm{Ca}_{2} \mathrm{Nb}_{3} \mathrm{O}_{10}\right]^{x-}$ is shown in Figure $\mathrm{S} 1$. This material is available free of charge via the Internet at http://pubs.acs.org.

\section{References and Notes}

(1) Domen, K.; Yoshimura, J.; Sekine, T.; Tanaka, A.; Onishi, T. Catal. Lett. 1990, 4, 339.

(2) Domen, K. Water Photolysis by Layered Compounds. In Photocatalysis Science and Technology; Kaneko, M., Okura, I., Eds.; Springer: New York, 2002; pp 261

(3) Takata, T.; Furumi, Y.; Shinohara, K.; Tanaka, A.; Hara, M.; Kondo, J. N.; Domen, K. Chem. Mater. 1997, 9, 1063.

(4) Zou, Z. G.; Ye, J. H.; Sayama, K.; Arakawa, H. Nature (London, U.K.) 2001, 414, 625. 571.

(5) Kudo, A.; Kato, H.; Nakagawa, S. J. Phys. Chem. B 2000, 104,

(6) Shimizu, K.; Tsuji, Y.; Hatamachi, T.; Toda, K.; Kodama, T.; Sato, M.; Kitayama, Y. Phys. Chem. Chem. Phys. 2004, 6, 1064.

(7) Domen, K.; Kondo, J. N.; Hara, M.; Takata, T. Bull. Chem. Soc. Jpn. 2000, 73, 1307.

(8) Kato, H.; Kudo, A. Catal. Lett. 1999, 58, 153.

(9) Takata, T.; Tanaka, A.; Hara, M.; Kondo, J. N.; Domen, K. Catal. Today 1998, 44, 17.

(10) Maeda, K.; Teramura, K.; Lu, D. L.; Takata, T.; Saito, N.; Inoue, Y.; Domen, K. Nature (London, U.K.) 2006, 440, 295.

(11) Tabata, S.; Ohnishi, H.; Yagasaki, E.; Ippommatsu, M.; Domen, K. Catal. Lett. 1994, 28, 417.

(12) Kudo, A.; Sayama, K.; Tanaka, A.; Asakura, K.; Domen, K.; Maruya, K.; Onishi, T. J. Catal. 1989, 120, 337.

(13) Sayama, K.; Tanaka, A.; Domen, K.; Maruya, K.; Onishi, T. J. Phys. Chem. 1991, 95, 1345.

(14) Kim, Y. I.; Atherton, S. J.; Brigham, E. S.; Mallouk, T. E. J. Phys. Chem. 1993, 97, 11802.

(15) Takata, T.; Shinohara, K.; Tanaka, A.; Hara, M.; Kondo, J. N.; Domen, K. J. Photochem. Photobiol., A 1997, 106, 45.

(16) Saupe, G. B.; Mallouk, T. E.; Kim, W.; Schmehl, R. H. J. Phys. Chem. B 1997, 101, 2508.

(17) Ebina, Y.; Sasaki, T.; Harada, M.; Watanabe, M. Chem. Mater. 2002, 14, 4390.

(18) Kato, H.; Asakura, K.; Kudo, A. J. Am. Chem. Soc. 2003, 125, 3082 .

(19) Shimizu, K.; Itoh, S.; Hatamachi, T.; Kodama, T.; Sato, M.; Toda, K. Chem. Mater. 2005, 17, 5161.

(20) Ebina, Y.; Sakai, N.; Sasaki, T. J. Phys. Chem. B 2005, 109, 17212

(21) Compton, O. C.; Carroll, E. C.; Larsen, D. S.; Osterloh, F. E. J. Phys. Chem. C 2007, 111, 14589-14592.

(22) Schaak, R. E.; Mallouk, T. E. Chem. Mater. 2002, 14, 1455.

(23) Kim, J. Y.; Osterloh, F. E.; Hiramatsu, H.; Dumas, R. K.; Liu, K. J. Phys. Chem. B 2005, 109, 11151.

(24) Kim, J. Y.; Osterloh, F. E. J. Am. Chem. Soc. 2006, 128, 3868.

(25) Kim, J. Y.; Hiramatsu, H.; Osterloh, F. E. J. Am. Chem. Soc. 2005 $127,15556$. 
(26) Furube, A.; Shiozawa, T.; Ishikawa, A.; Wada, A.; Domen, K.; Hirose, C. J. Phys. Chem. B 2002, 106, 3065.

(27) Tamaki, Y.; Furube, A.; Murai, M.; Hara, K.; Katoh, R.; Tachiya, M. J. Am. Chem. Soc. 2006, 128, 416.

(28) Tamaki, Y.; Furube, A.; Murai, M.; Hara, K.; Katoh, R.; Tachiya, M. Phys. Chem. Chem. Phys. 2007, 9, 1453.

(29) Izawa, K.; Yamada, T.; Unal, U.; Ida, S.; Altuntasoglu, O.; Koinuma, M.; Matsumoto, Y. J. Phys. Chem. B 2006, 110, 4645.

(30) Jacobsen, A. J.; Johnson, J. W.; Lewandowski, J. T. Inorg. Chem. 1985, 24, 3727

(31) Schaak, R. E.; Mallouk, T. E. Chem. Mater. 2000, 12, 2513.

(32) Han, Y. S.; Park, I.; Choy, J. H. J. Mater. Chem. 2001, 11, 1277.

(33) Wiegel, M.; Hamoumi, M.; Blasse, G. Mater. Chem. Phys. 1994 $36,289$.

(34) Pankove, J. I. Optical Processes in Semiconductors; Dover Publishing, Inc.: New York, 1975

(35) Kudo, A.; Kaneko, E. J. Mater. Sci. Lett. 1997, 16, 224.

(36) Rothenberger, G.; Moser, J.; Gratzel, M.; Serpone, N.; Sharma, D.

K. J. Am. Chem. Soc. 1985, 107, 8054

(37) Bahnemann, D. W.; Hilgendorff, M.; Memming, R. J. Phys. Chem. B 1997, 101, 4265 .

(38) Tamaki, Y.; Furube, A.; Katoh, R.; Murai, M.; Hara, K.; Arakawa, H.; Tachiya, M. C. R. Chim. 2006, 9, 268.

(39) Serpone, N.; Lawless, D.; Khairutdinov, R.; Pelizzetti, E. J. Phys. Chem. 1995, 99, 16655.

(40) Cavaleri, J. J.; Colombo, D. P.; Bowman, R. M. J. Phys. Chem. B 1998, 102, 1341.

(41) Colombo, D. P.; Roussel, K. A.; Saeh, J.; Skinner, D. E.; Cavaleri, J. J.; Bowman, R. M. Chem. Phys. Lett. 1995, 232, 207.

(42) Barzykin, A. V.; Tachiya, M. J. Phys. Chem. B 2002, 106, 4356.

(43) Grela, M. A.; Colussi, A. J. J. Phys. Chem. 1996, 100, 18214.
(44) Oneil, M.; Marohn, J.; McLendon, G. J. Phys. Chem. 1990, 94, 4356.

(45) Xu, F. F.; Ebina, Y.; Bando, Y.; Sasaki, T. J. Phys. Chem. B 2003, 107,9638

(46) Yoshihara, T.; Katoh, R.; Furube, A.; Tamaki, Y.; Murai, M.; Hara, K.; Murata, S.; Arakawa, H.; Tachiya, M. J. Phys. Chem. B 2004, 108 , 3817.

(47) Yoshihara, T.; Tamaki, Y.; Furube, A.; Murai, M.; Hara, K.; Katoh, R. Chem. Phys. Lett. 2007, 438, 268

(48) Bahnemann, D.; Henglein, A.; Lilie, J.; Spanhel, L. J. Phys. Chem. 1984, 88, 709 .

(49) Zhang, Z. B.; Wang, C. C.; Zakaria, R.; Ying, J. Y. J. Phys. Chem. B 1998, 102, 10871 .

(50) Skinner, D. E.; Colombo, D. P.; Cavaleri, J. J.; Bowman, R. M. J. Phys. Chem. 1995, 99, 7853.

(51) Colombo, D. P.; Bowman, R. M. J. Phys. Chem. 1996, 100, 18445

(52) Miyamoto, N.; Yamada, Y.; Koizumi, S.; Nakato, T. Angew. Chem., Int. Ed. 2007, 46, 4123 .

(53) Chen, T.; Feng, Z.; Wu, G.; Shi, J.; Ma, G.; Ying, P.; Li, C. J. Phys. Chem. B 2007, 111, 8005 .

(54) Klimov, V. I. Semiconductor and Metal Nanocrystals: Synthesis and Electronic and Optical Properties; Marcel Dekker, Inc.: New York, 2004.

(55) Burda, C.; Green, T. C.; Link, S.; El-Sayed, M. A. J. Phys. Chem B 1999, 103, 1783.

(56) Burda, C.; El-Sayed, M. A. Pure Appl. Chem. 2000, 72, 165.

(57) Landes, C. F.; Braun, M.; El-Sayed, M. A. J. Phys. Chem. B 2001, 105,10554

(58) Landes, C.; Burda, C.; Braun, M.; El-Sayed, M. A. J. Phys. Chem. B 2001, 105, 2981. 